\title{
Reacciones psoriasiformes paradójicas secundarias a terapias biológicas con anti-TNF-alfa
}

\section{Paradoxical psoriasiform reactions due to anti-TNF-alpha biologic treatment}

\author{
Luciana L. Tirelli ${ }^{1,2}$, Paula C. Luna ${ }^{1,2}$, Juan J. Solé1,2, Alejandra A. Panizzardi ${ }^{1,2}$, Bruno Ferrari ${ }^{2}$, Luis \\ D. Mazzuoccolo ${ }^{2}$, Cristina Echeverría ${ }^{2}$, María T. González Baschiera ${ }^{2}$, Federico Barrera ${ }^{1}$, Andrés \\ Label $^{1}$, Marina Mónaco ${ }^{1}$ y Margarita Larralde ${ }^{1}$
}

\begin{abstract}
RESUMEN
Las reacciones psoriasiformes paradójicas son consecuencia del uso prolongado y cada vez mayor de los distintos tratamientos biológicos; tal es el caso de los anti-TNF-alfa. Las reacciones paradójicas consisten en la exacerbación o en la aparición de un nuevo síntoma o de otra enfermedad que, por lo general, mejoran con el empleo de agentes biológicos como los anti-TNF-alfa. Estos fármacos presentan múltiples indicaciones en relación con distintas enfermedades inflamatorias; por lo tanto, es de suma relevancia que todos los especialistas que los utilizan estén al tanto de esa entidad.
\end{abstract}

El propósito de este trabajo fue observar y describir las características demográicas y clínicas de un grupo de pacientes con psoriasis paradójica y compararlas con las publicadas en la literatura.

Palabras clave: psoriasis, reacción psoriasiforme paradójica, anti-TNF-alfa, tratamiento biológico.

Dermatol. Argent. 2020, 26 (2): 63-67

\begin{abstract}
As a consequence of the advance in different medical treatments, like with the use of biologic drugs such as anti-TNF-alpha, there can be seen paradoxical psoriasiform reactions. The paradoxical reactions consist of the exacerbation or the appearance of a new symptom or another disease that, generally, improve with the use of biological agents such as anti-TNF-alpha, drugs have several indications related to the treatment of different inflammatory pathologies, hence, it is crucial that every specialist who prescribes them are aware of this secondary effect.
\end{abstract}

The aim of this article was to observe and describe the demographic and clinical characteristics of a group of patients with paradoxical psoriasiform reactions; and to compare them with the literature.

Key words: psoriasis, paradoxical psoriasiform reactions, anti-TNF- alpha, biologic treatment.

Dermatol. Argent. 2020, 26 (2): 63-67

\footnotetext{
'Servicio de Dermatología, Hospital Alemán, Ciudad Autónoma de Buenos Aires, Argentina

${ }^{2}$ Grupo ECHO Psoriasis Argentina
}

Contacto del autor: Luciana Tirelli E-mail: lucianatirelli@yahoo.com.ar Fecha de trabajo recibido: 3/10/2019 Fecha de trabajo aceptado: 9/6/2020 Conflicto de interés: los autores declaran que no existe conflicto de interés.

\section{INTRODUCCION}

Los fármacos biológicos anti-TNF-alfa se han utilizado durante mucho tiempo para el tratamiento de las formas severas no solo de la psoriasis, sino también de múltiples patologías inflamatorias. Estos medicamentos se han publicado como un excelente recurso para el tratamiento de la psoriasis severa. Llamativamente, en algunos casos son el gatillo de reacciones psoriasiformes paradójicas y de ahí su nombre. El mecanismo por el cual se desencadena tal fenómeno no está esclarecido en su totalidad, pero se estima que resulta de un 
desequilibrio en las vías inflamatorias a favor del interferón alfa. Esas reacciones ocurren en un 5\% de los pacientes que utilizan anti-TNF-alfa, cualquiera que sea la patología que motivó su indicación. Todos los fármacos de su clase pueden desencadenar reacciones adversas cutáneas. De modo característico, se describe un empeoramiento del tipo de psoriasis preexistente, un cambio morfológico en la forma clínica (en caso de presentar antecedentes de psoriasis) o la aparición de un cuadro psoriasiforme de novo. Si bien este efecto adverso se puede observar dentro de un rango variable de tiempo desde el inicio del tratamiento con estos fármacos, la mayoría de los casos se producen durante el primer año de su administración.

Consideramos de interés, en primer lugar, aportar nuestra serie de casos a la literatura médica nacional, ya que no existen comunicaciones previas acerca de este tema. Asimismo, dado el amplio rango de patologías que son tratadas con este grupo de fármacos, creemos necesario difundir nuestra experiencia no solo a los colegas de nuestra especialidad, sino a toda la comunidad

\begin{tabular}{|c|c|c|c|c|c|c|c|c|c|}
\hline Paciente & Sexo & $\begin{array}{l}\text { Edad } \\
\text { (años) }\end{array}$ & $\begin{array}{l}\text { Comorbili- } \\
\text { dad }\end{array}$ & $\begin{array}{c}\text { Antecedente } \\
\text { familiar } \\
\text { de psoriasis }\end{array}$ & $\begin{array}{l}\text { Antece- } \\
\text { dente } \\
\text { personal }\end{array}$ & $\begin{array}{l}\text { Forma clínica } \\
\text { de psoriasis }\end{array}$ & $\begin{array}{l}\text { Tratamiento } \\
\text { biológico previo }\end{array}$ & $\begin{array}{c}\text { Tratamiento } \\
\text { actual }\end{array}$ & $\begin{array}{l}\text { Tiempo de } \\
\text { latencia } \\
\text { (meses) }\end{array}$ \\
\hline 1 & $\mathrm{~F}$ & 49 & & & EC & Vulgar/gotas & $\begin{array}{l}\text { Infliximab/certoli- } \\
\text { zumab }\end{array}$ & & 2 \\
\hline 2 & M & 33 & TBQ & & EA & $\begin{array}{l}\text { Pustulosa } \\
\text { palmoplantar }\end{array}$ & Certolizumab & Secukinumab & 9 \\
\hline 3 & M & 84 & HTA, IC & & CU & Eritrodérmica & Adalimumab & Muerte* & 1 \\
\hline 4 & $\mathrm{~F}$ & 34 & TBQ & & CU & Palmoplantar & Adalimumab & Fototerapia & 12 \\
\hline 5 & $\mathrm{~F}$ & 27 & & & $\mathrm{EC}$ & Vulgar & $\begin{array}{l}\text { Infliximab/adalimu- } \\
\text { mab/certolizumab }\end{array}$ & $\begin{array}{l}\text { Se adicionó trata- } \\
\text { miento tópico }\end{array}$ & $60 / 12$ \\
\hline 6 & $\mathrm{~F}$ & 44 & & & EC & Gotas & Certolizumab & $\begin{array}{l}\text { Se adicionó } \\
\text { metotrexato }\end{array}$ & 4 \\
\hline 7 & M & 45 & & & PV & Gotas & Adalimumab & $\begin{array}{l}\text { Sin tratamiento } \\
\text { actualmente }\end{array}$ & 36 \\
\hline 8 & $\mathrm{~F}$ & 53 & & & $A R$ & Palmoplantar & Adalimumab & $\begin{array}{l}\text { Tofacitinib y } \\
\text { fototerapia }\end{array}$ & 12 \\
\hline 9 & $\mathrm{~F}$ & 72 & Asma & & EC & Vulgar & Adalimumab & Metotrexato & 18 \\
\hline 10 & $F$ & 52 & & & CU & Palmoplantar & Adalimumab & $\begin{array}{l}\text { Se adicionó trata- } \\
\text { miento tópico }\end{array}$ & 24 \\
\hline 11 & $M$ & 30 & & $\begin{array}{l}\text { Familiar de } \\
\text { segundo grado }\end{array}$ & HS & Sebopsoriasis & Adalimumab & $\begin{array}{l}\text { Se adicionó trata- } \\
\text { miento tópico }\end{array}$ & 9 \\
\hline 12 & M & 16 & & & $\mathrm{ECyCU}$ & Vulgar/gotas & Infliximab & Adalimumab & 18 \\
\hline 13 & $\mathrm{~F}$ & 27 & & & $\mathrm{EC}$ & Vulgar & Adalimumab & $\begin{array}{l}\text { Se adicionó trata- } \\
\text { miento tópico }\end{array}$ & 6 \\
\hline 14 & $\mathrm{~F}$ & 52 & $\begin{array}{l}\text { TBQ, HTA, } \\
\text { hipotiroidis- } \\
\text { mo, EHNA }\end{array}$ & & PV & $\begin{array}{l}\text { Vulgar/palmo- } \\
\text { plantar }\end{array}$ & Adalimumab & Ustekinumab & 3 \\
\hline
\end{tabular}

médica, a fin de tomar conductas terapéuticas multidisciplinarias y acertadas para cada caso en particular.

\section{SERIE DE CASOS}

Se conformó una cohorte de pacientes con diagnóstico de reacciones psoriasiformes paradójicas secundarias al tratamiento con anti-TNF-alfa atendidos en el Hospital Alemán durante los últimos 5 años (2014-2019). Otros casos fueron aportados por un grupo de estudio de pacientes con psoriasis (grupo ECHO) durante el mismo período. ECHO Argentina está conformado por un grupo de dermatólogos (223) distribuidos en las distintas provincias del país, quienes llevan a cabo encuentros mensuales a través de una plataforma virtual (ZOOM), con la finalidad de presentar casos clínicos de psoriasis y debatir distintos aspectos de interés.

Se reunieron 14 pacientes con diagnóstico de reacción psoriasiforme paradójica durante el tratamiento con anti-TNF-alfa, por distintas patologías inflamatorias preexistentes (Tabla 1). Se evaluaron diferentes 
variables como edad, sexo, comorbilidades, antecedentes familiares, edad al inicio y al diagnóstico, demora en la realización de este, forma clínica de las lesiones, presencia de compromiso articular y tratamientos anteriores e instaurados en el servicio de dermatología (o médico tratante).

De los 14 pacientes, un varón era pediátrico y el resto, pacientes adultos (mayores de 18 años). En nuestra serie hubo un franco predominio del sexo femenino (9 de 14 pacientes) por sobre el masculino. Llamativamente, un solo paciente presentaba antecedente familiar (familiar de segundo grado) de psoriasis, lo cual coincide con la literatura. Asimismo, tampoco se registró demora en el diagnóstico; es decir, una vez que cada paciente presentó la reacción psoriasiforme paradójica, la consulta con un profesional de la salud se realizó de inmediato. Interpretamos este hecho como consecuencia del seguimiento estrecho del paciente por parte del sistema de salud y su equipo médico tratante.

$\mathrm{Al}$ igual que lo publicado en la literatura, en nuestra serie las formas clínicas de psoriasis más observadas fueron la vulgar, seguida de la palmoplantar. Ningún paciente presentó compromiso articular como manifestación paradójica (Fotos1-4).

En nuestra experiencia, la comorbilidad referida con mayor frecuencia fue el tabaquismo.

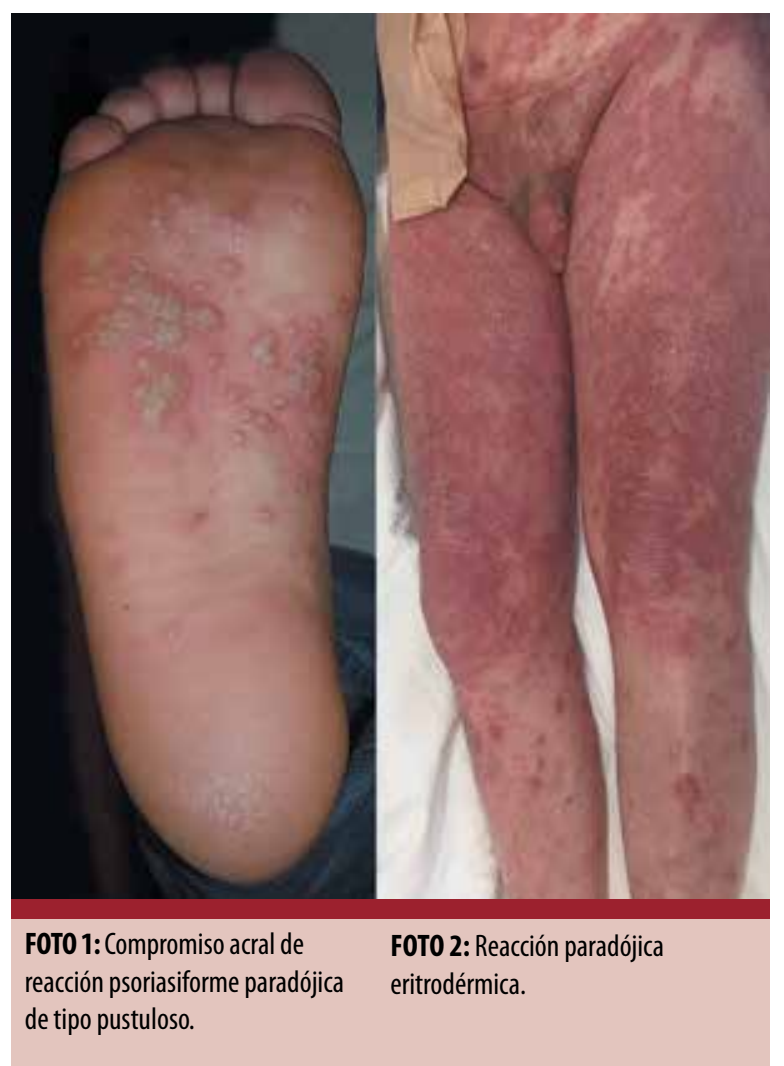

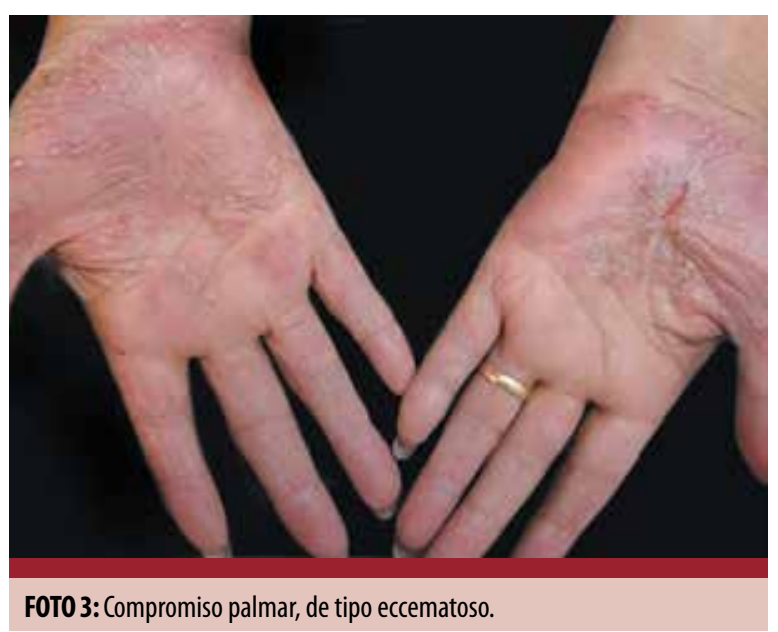

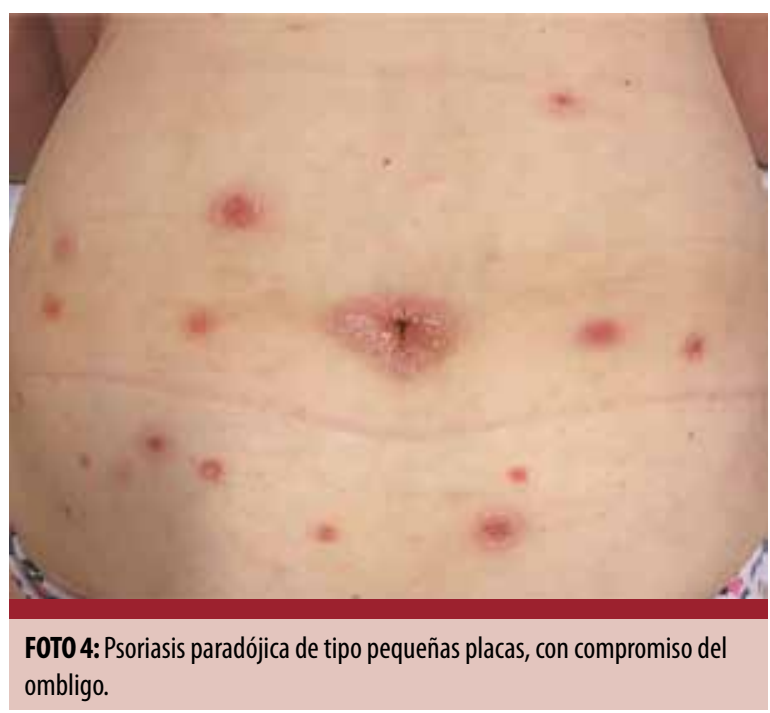

\section{COMENTARIOS}

La psoriasis es una patología inflamatoria, multifactorial, de curso crónico, que afecta a un 3\% de la población mundial. Dentro de este porcentaje se calcula que alrededor de un $40 \%$ de los casos corresponderían a formas moderadas a severas. En este subgrupo de formas clínicas, en el que se utilizan distintos tratamientos sistémicos, los anti-TNF-alfa son una alternativa terapéutica. Estos fármacos se prescriben desde hace varias décadas, no solo para la psoriasis, sino también en el tratamiento de las formas severas de múltiples enfermedades inflamatorias. Como consecuencia del amplio uso de este grupo farmacológico, se han comenzado a observar sus efectos adversos; el que aquí nos concierne es la reacción psoriasiforme paradójica. Se denomina de esta forma, ya que los anti-TNF-alfa son un tratamiento contra la psoriasis severa, si bien en algunas ocasiones pueden desencadenarla ${ }^{1,2}$. En los casos en los que se conoce el antecedente de psoriasis, estas reacciones paradójicas se pueden manifestar 
como exacerbación del cuadro preexistente o como un cambio en su forma clínica, mientras que en aquellos casos en los que se carece del antecedente, se puede apreciar su aparición de novo. Las formas clínicas de reacciones psoriasiformes paradójicas más comunicadas son las del tipo placa y la palmoplantar ${ }^{3,4}$.

En relación con nuestra serie de pacientes, solo 2 de ellos (2/14) tenían el antecedente de psoriasis y esta fue la indicación que motivó el inicio del tratamiento con anti-TNF. De los $12(12 / 14)$ pacientes restantes, $6(6 / 14)$ presentaban diagnóstico de enfermedad de Crohn, 4 (4/14) de colitis ulcerosa, uno (1/14) de espondilitis anquilosante, uno (1/14) de artritis reumatoide y uno (1/14) de hidradenitis supurativa, por lo que recibieron el tratamiento biológico. Estas proporciones se relacionan con lo comunicado en la bibliografía y, en la mayoría, provienen de los servicios de gastroenterología. Se destaca que solo un paciente (1/14) presentaba antecedentes familiares de psoriasis (familiar de segundo grado); esta baja asociación (a diferencia de lo observado con la psoriasis primaria) se relaciona con lo referido en la literatura. Esto no excluye el hecho de presentar susceptibilidad genética para desarrollar reacciones paradójicas inducidas por anti-TNF5.

Según las distintas series, se calcula que 1 de cada 4 pacientes en tratamiento con anti-TNF-alfa sufrirá algún efecto adverso cutáneo asociado ${ }^{2,3}$. Cabe destacar que las reacciones paradójicas son un efecto adverso de clase, es decir, cualquier anti-TNF puede desencadenarlo; incluso si se cambia un fármaco por otro del mismo grupo, se han comunicado rebrotes con las mismas características ${ }^{3-7}$. En relación con el grupo de pacientes de nuestra cohorte, este fenómeno se pudo observar en 2 de ellos. Uno había recibido infliximab y certolizumab; el otro, adalimumab y certolizumab. En ambos se reprodujeron las lesiones paradójicas a pesar del cambio de la medicación.

El tiempo de latencia desde la introducción del tratamiento biológico hasta el desarrollo de los efectos adversos es variable. Se puede observar su aparición a los pocos días de iniciado, o también después de varios meses o años ${ }^{3}$. Algunos autores proponen una media de 10 meses $^{4,7}$. Según nuestra experiencia, este intervalo fue bastante amplio: desde un mes hasta 60 meses desde el inicio del anti-TNF. De todas formas, la mayoría de los pacientes (9/14) manifestaron la reacción paradójica dentro del primer año de tratamiento y 12 pacientes (12/14) lo hicieron dentro de los 2 primeros ańos.

La fisiopatología de estas reacciones paradójicas aún no se ha dilucidado en su totalidad. Existen mu- chas hipótesis al respecto; la más aceptada es aquella en la que se postula que debido a la supresión de la vía proinflamatoria dependiente de TNF-alfa, se sobreestimula la síntesis de interferón alfa (IFN-alfa) por parte de las células dendríticas. A su vez, se plantea que al bloquear la vía TNF podrían estimularse exageradamente los linfocitos $\mathrm{T}$ epidérmicos, lo que induce la proliferación de queratinocitos y desencadena los cuadros psoriasiformes. Otro aspecto relacionado, consecuencia del antagonismo del TNF-alfa, es la inducción de la sobreexpresión de los receptores de quimioquinas como CXCR3, que promueve la migración de linfocitos $\mathrm{T}$ autorreactivos hacia la epidermis, al igual que en la psoriasis ${ }^{4,8,9}$.

En nuestra serie, el anti-TNF que con mayor frecuencia produjo reacciones paradójicas fue el adalimumab. En relación con ello, cabe destacar que la mayoría de los pacientes provenían derivados de distintos servicios de gastroenterología. Estos especialistas utilizan como tratamiento contra la enfermedad inflamatoria intestinal solo el adalimumab y el infliximab.

Por último, un aspecto aún no esclarecido es la conducta por tomar frente a las reacciones paradójicas. Hay múltiples posturas según los diferentes autores y las especialidades involucradas. Hay quienes proponen que, frente a un efecto secundario de este tipo, se debe rotar el tratamiento biológico por otro con un mecanismo de acción alternativo, mientras que otros autores proponen evaluar el estado de la patología subyacente que motivó la indicación del anti-TNF y, en caso de encontrarse controlada, adicionar otro tipo de tratamiento para controlar la reacción paradójica ${ }^{10,11}$. En contraposición, algunos autores también proponen rotar el fármaco en cuestión por otro anti-TNF y, si se repite la reacción paradójica con un segundo anti-TNF, cambiar de tratamiento con otro mecanismo farmacológico diferente ${ }^{12}$.

\section{CONCLUSIÓN}

A partir del análisis de estos datos se pudo concluir que la mayoría de los pacientes fueron mujeres, no hubo demora desde el inicio de las lesiones hasta el diagnóstico de la reacción psoriasiforme paradójica, solo un paciente refirió antecedentes familiares de psoriasis (familiar de segundo grado), las formas clínicas de psoriasis vulgar seguida de la forma palmoplantar fueron las más observadas dentro del grupo en estudio, la comorbilidad asociada con mayor frecuencia fue el tabaquismo, el fármaco más vinculado a este efecto adverso fue el adalimumab y la mayoría de los pacientes presentaron un tiempo de latencia hasta la aparición de la reacción paradójica de 1-12 meses, al igual que 
lo establecido en la literatura. Nuestras observaciones no defieren de las características comunicadas en la bibliografía referida a este tema. Consideramos de

\section{BIBLIOGRAFÍA}

1. Wendling D, Prati C. Paradoxical effects of anti-TNF-a agents in inflammatory diseases. Expert Rev Clin Inmunol 2014;10:159-169.

2. Segaert S, Hermans C. Clinical signs, pathophysiology and management of cutaneous side effects of anti-tumor necrosis factor agents. Am J Clin Dermatol 2017;18:771-787.

3. Fréling E, Baumann C, Cuny JF, Bigard MA, et ál. Cumulative incidence of, risk factors for, and outcome of dermatological complications of anti-TNF therapy in inflammatory bowel disease: A 14-year experience. Am J Gastroenterol 2015;110:1186-1196.

4. Joyau C, Veyrac G, Dixneuf V, Jolliet P. Anti-tumour necrosis factor alpha therapy and increased risk of de novo psoriasis: is it really a paradoxical side effect? Clin Exp Rheumatol 2012;30:700-706.

5. Iborra $M$, Beltrán B, Bastida G, Aguas $M$, et ál. Infliximab and adalimumab-induced psoriasis in Crohn's disease: a paradoxical side effect. J Crohns Colitis 2011;5:157-161.

6. Nguyen K, Vleugels RA, Velez NF, Merola J, et ál. Psoriasiform reactions to anti-tumour necrosis factor a therapy. J Clin interés aportar esta serie de pacientes a la comunidad científica, ya que no existen datos nacionales relacionados con este trastorno.

Rheumatol 2013;19:377-381.

7. Navarro R, Daudén E. Clinical management of paradoxical psoriasiform reactions during TNF-a therapy. Actas Dermosifiliogr 2014, 105:752-761.

8. Sfikakis PP, lliopoulos A, Elezoglou A, Kittas C, et ál. Psoriasis induced by anti-tumor necrosis factor therapy: A paradoxical adverse reaction. Arthritis Rheum 2005;52:2513-2518.

9. Conrad C, Di Domizio J, Mylonas A, Belkhodja C, et ál. TNF blockade induces a dysregulated type I interferon response without autoimmunity in paradoxical psoriasis. Nat Commun 2018;1-11.

10. Ko JM, Gottlieb AB, Kerbleski JF. Induction and exacerbation of psoriasis with TNF-blockade therapy: a review and analysis of 127 cases. J Dermatolog Treat 2009;20:100-108.

11. Groth $\mathrm{D}$, Perez M, Treat JR, Castelo-Soccio L, et ál. Tumor necrosis factor-a inhibitor-induced psoriasis in juvenile idiopathic arthritis patients. Pediatr Dermatol 2019;36:613-617.

12. Garcovich S, De Simone C, Genovese G, Benti E, et ál. Paradoxical skin reactions to biologics in patients with rheumatologic disorders. Front Pharmacol 2019;10:1-8.

\section{DERMATÓLOGOS JÓVENES}

\section{Elección múltiple: pápulas y vesículas generalizadas}

Ana Pereyra y Mariana Martínez

Servicio de Dermatología, Hospital General de Agudos Dr. Enrique Tornú, Ciudad Autónoma de Buenos Aires, Argentina

Una paciente de 26 años, sin antecedentes personales ni familiares de relevancia, consultó por la presencia de una dermatosis generalizada polimorfa, con extensión cefalocaudal, asociada a fiebre, de 3 días de evolución. En el examen físico se observaban múltiples pápulas, vesículas y pústulas, algunas con costra necrótica central, pruriginosas, que afectaban el cuero cabelludo, la región retroauricular, la cara, el tronco, los miembros superiores e inferiores, con compromiso de las palmas y las plantas.

\section{1) ¿Cuál es el diagnóstico más probable? \\ a. Prurigo por picaduras de insectos. \\ b. Papulosis linfomatoide. \\ c. Varicela. \\ d. Pitiriasis liquenoide y varioliforme aguda. e. Sífilis secundaria.}

2) ¿Qué estudio solicitaría para realizar el diagnóstico?

a. VDRL cuantitativa.

b. Biopsia de piel para estudio histopatológico. c. IgM e lgG para virus de la varicela zóster por ELISA. d. El diagnóstico es clínico, no realizaría ningún estudio complementario.

e. Prueba de Tzanck de la base de una vesícula.

3) Si se realizara biopsia, ¿qué esperaría encontrar en el estudio histopatológico?

a. Infiltrado triangular en cuña con células tumorales $C D$ 30+ entremezcladas con células inflamatorias.

b. Vesícula intraepidérmica, acantólisis, degeneración reticular con células gigantes multinucleadas con inclusiones intranucleares acidófilas, edema y vasculitis en la dermis.

c. Paraqueratosis, espongiosis, degeneración vacuolar de la membrana basal con exocitosis de linfocitos y eritrocitos, queratinocitos necróticos. En la dermis, edema con infiltrado linfocitario perivascular. Predominio de linfocitos TCD8.

d. Hiperplasia psoriasiforme, exocitosis de linfocitos, pústulas espongiformes y paraqueratosis. En la dermis, infiltrados linfohistiocitarios perivasculares o perianexiales.
FOTOS 1 y 2: Múltiples vesículas y pústulas, algunas con una costra necrótica central, localizadas en la cara, el dorso y la cara posterior de los miembros superiores.

e. Edema, vasodilatación de la dermis con infiltrados linfocitarios y abundantes eosinófilos.

4) ¿Qué tratamiento considera de primera línea? a. Aciclovir $800 \mathrm{mg}$ cada 4 horas, 5 veces por día durante 5 a 7 días.

b. Antihistamínicos por vía oral y corticosteroides tópicos.

c. Minociclina $100 \mathrm{mg}$ cada 12 horas durante 14 días.

d. Una dosis intramuscular de penicilina $G$ benzatínica 2.400.000 Ul.

e. Fototerapia.

Las respuestas en la página 86 